Diabetologia (1995) 38: 129-134

\title{
Effects of anti-oxidant treatment on sciatic nerve dysfunction in streptozotocin-diabetic rats; comparison with essential fatty acids
}

\author{
Ç. Karasu ${ }^{1}$, M.Dewhurst ${ }^{2}$, E.J.Stevens ${ }^{2}$, D.R. Tomlinson ${ }^{1,2}$ \\ ${ }^{1}$ Department of Pharmacology, Queen Mary and Westfield College, University of London, London, UK \\ ${ }^{2}$ William Harvey Research Institute, Queen Mary and Westfield College, University of London, London, UK
}

\begin{abstract}
Summary In Study 1 , the effects of treatment of streptozotocin-diabetic rats with the antioxidants, probucol or vitamin E were compared. Untreated diabetic rats showed a reduction of $45 \%(p<0.01)$ in nerve laser Doppler flux, which was used as an index of nerve blood flow. In diabetic rats treated with either probucol or vitamin E nerve Doppler flux was reduced by only 13 or $16 \%$, respectively $(p<0.01$ for either compared to untreated diabetic rats). A second study examined the effects of treatment with evening primrose oil either alone or in combination with probucol. Reduced nerve Doppler flux was reproduced in untreated diabetic rats $(47 \% ; p<0.01)$. In parallel diabetic groups, nerve Doppler flux was reduced by only $14 \%$ with evening primrose oil alone and by $8 \%$ with evening primrose oil plus probucol (both $p<0.01$ vs untreated diabetic rats). Both treatments were also associated with marked
\end{abstract}

attenuation of motor and sensory nerve conduction velocity deficits. Measurements on plasma from rats showed normalisation of triglyceride levels by probucol treatment without an effect on those of cholesterol in Study 1. In Study 2, the converse was true for evening primrose oil treatment, whilst the combined treatment lowered both plasma triglycerides and cholesterol. This work indicates similar effects of antioxidants and evening primrose oil against reduced nerve Doppler flux and conduction velocity in diabetic rats, with dissimilar actions on plasma triglycerides and cholesterol. [Diabetologia (1995) 38: 129134]

Key words Diabetes mellitus, Doppler flux, nerve conduction, neuropathy, cholesterol, triglycerides, rat, streptozotocin.
Vascular insufficiency, resulting in endoneurial ischaemia, may make an important aetiological contribution to nerve conduction deficits in the early stages of experimental diabetes $[1,2]$. This implication of ischaemia in the causation of acute conduction defects is supported by the finding that nerve blood flow is reduced in the sciatic nerves of diabetic rats $[3,4]$ and that many of the pharmacological interventions, which prevent nerve conduction abnormalities associated with diabetes, have the unifying property

Received: 28 January 1994 and in revised form: 9 August 1994

Corresponding author: Professor D. R. Tomlinson, Department of Pharmacology, Queen Mary and Westfield College, Mile End Road, London E1 4NS, UK

Abbreviations: STZ, streptozotocin. of improving nerve blood flow [4, 5]. Indeed, previous studies have demonstrated that the slowed nerve conduction can be attenuated or prevented by treatment of diabetic rats with several vasodilators of differing pharmacological modes of action [6-8]. We have previously shown that the reduced sciatic nerve laser Doppler flux (an index of nerve blood flow) in diabetic rats, can also be prevented by treatment with essential fatty acids [5], possibly via production of vasodilator prostaglandins [9].

There is increasing evidence of free radical-derived oxidative stress in diabetes together with an involvement in the development of complications [10-13]. The source of oxygen-derived free radicals in diabetes may be auto-oxidation of glucose, intermediate products of cyclooxygenase catalysis or intracellular production from mitochondria or both [14]. Plasma 
lipid peroxides have been reported to be elevated in diabetic humans particularly in associated with poorly-controlled plasma glucose [15], with the potential to cause vascular abnormalities, ischaemia and a markedly increased risk of angiopathy [11, 16-18]. Streptozotocin(STZ)-diabetic rats also sustain increased levels of plasma lipid peroxidation products, which can be reduced by dietary probucol [19]. As a result of diabetes-induced oxidative stress, the vasorelaxant capacity of vascular endothelium is significantly attenuated, which has been linked with reduced prostacyclin and nitric oxide release $[11,20,21]$, possibly resulting in decreased local blood flow. Previous reports have indicated that probucol has a protective effect against endothelial cell dysfunction caused by elevated glucose [22]. Recently, it has also been shown that some nerve dysfunctions in experimental diabetes are restored by treatment with probucol [23], as well as with the anti-oxidant butylated hydroxytoluene [24] and glutathione [25]. Accordingly, we designed the first part of the present study to investigate the effects of treatment of diabetic rats with the anti-oxidants probucol or vitamin $\mathrm{E}$ on sciatic nerve laser Doppler flux. Since the double bonds of unsaturated essential fatty acids are readily oxidised, we also compared the influences of dietary low-dose evening primrose oil supplementation, either alone or with probucol on nerve laser Doppler flux and motor and sensory nerve conduction velocity.

\section{Materials and methods}

Experimental organisation. Two experiments (Study 1 and Study 2) were carried out on male Wistar rats of starting weight $250-300 \mathrm{~g}$ and age range 8-12 weeks (Charles River (UK) Ltd., Margate, Kent, UK). In both experiments the animals were randomly assigned to four experimental groups. Rats were maintained under standard housing conditions with normal rat chow (see below) and water available ad libitum. Age-matched non-diabetic rats were employed as onset control groups. For induction of diabetes, rats were fasted overnight and at 09.00 hours the following morning were given an intraperitoneal injection of STZ (Sigma, Poole, Dorset, UK; $50 \mathrm{mg} / \mathrm{kg}$, freshly dissolved in sterile $0.9 \%$ aqueous $\mathrm{NaCl}$ ). Two days later diabetes was verified by estimating hyperglycaemia on tail vein blood (Reflolux II; Boehringer Mannheim, Lewes, UK). All animals had a morning blood glucose concentration greater than $15 \mathrm{mmol} / \mathrm{l}$ and were included in the studies. Blood glucose was monitored at weekly intervals. One group of diabetic rats from each study was untreated for 4 5 weeks to act as diabetic controls. In both studies, the latter and the non-diabetic control rats were fed a powdered diet (RM No.1; Special Diet Services Ltd., Witham, Essex, UK) without any supplement. In Study 1, another diabetic group was provided with powdered diet containing probucol (Sigma) to comprise $1 \%$ of dietary weight. The third diabetic group was fed powdered diet containing vitamin $\mathrm{E}(1 \%$ weight/weight, Sigma). Vitamin E-containing diets were stored at $4{ }^{\circ} \mathrm{C}$. In Study 2 , in addition to the untreated diabetic group, groups of diabetic rats were treated with evening primrose oil alone $\left(1 \mathrm{ml} \cdot \mathrm{kg}\right.$ body weight ${ }^{-1} \cdot$ day $^{-1}$ by gavage; Efa- mol; Scotia Pharmaceuticals, Guildford, Surrey, UK) or with evening primrose oil (dose as above) plus a powdered diet containing probucol $(1 \% \mathrm{w} / \mathrm{w})$. Animals were maintained for a period of 4 to 5 weeks. Body weight was recorded at least once a week. Food and water consumption were measured daily over a 24 -h period for each cage of four rats, but data were transformed to give values $\cdot \mathrm{rat}^{-1} \cdot 24 \mathrm{~h}^{-1}$. Prior to the experiment, final body weights and blood glucose concentrations were measured by a glucose oxidase strip-operated reflectance meter (as above).

Sciatic nerve laser Doppler flux and conduction velocities. These were measured under the same non-recovery anaesthesia, which is described in detail elsewhere [26]. Briefly, the rats were anaesthetised with alphaxalone and alphadolone acetate (3 : 1 w/w; Saffan; Pitman-Moore Ltd, Uxbridge, UK), using an initial dose of $4 \mathrm{mg} / \mathrm{kg}$ followed by a continuous infusion of $12 \mathrm{mg} / \mathrm{h}$. Systemic arterial pressure and sciatic nerve laser Doppler flux were then measured exactly as described elsewhere [26]. Motor and sensory nerve latencies were subsequently measured. During these measurements nerve temperature was controlled by placement of a microthermocouple adjacent to the mid-femoral sciatic nerve, inserted via a small incision higher in the flank. The wound was closed around the thermocouple leads, which were connected to an electronic thermometer (Comark, Rustington, Sussex, UK). The left flank was warmed with an infra-red lamp until the sciatic nerve temperature was $37^{\circ} \mathrm{C}$. The left sciatic nerve was stimulated using a Maclab integral stimulator (AD Instruments Ltd., London, UK) first at the sciatic notch and then at the Achilles tendon by means of monopolar needle electrodes using a regular supra-maximal single pulse stimulus (duration $0.1 \mathrm{~ms}$ and voltage $10 \mathrm{~V}$ ). In response to each stimulus an interosseous electromyogram was recorded by needle electrodes, digitised and displayed using a Maclab and Macintosh personal computer via "Scope 3.2" software (AD Instruments). The temporal separations of the electromyograms obtained at the two stimulus positions were calculated and used for computation of motor nerve conduction velocity with reference to nerve length. The same stimuli also evoke second compound muscle action potentials (of reversed latencies) due to excitation of fast conducting afferents, which connect monosynaptically with $\alpha$-motoneurons. This latency difference was used to calculate sensory nerve conduction velocity for group Ia afferents.

At the end of the procedure the rat was exsanguinated from the right ventricle, to provide blood for assays as detailed below and the sciatic nerve used for conduction and Doppler flux was removed for measurement of nerve length to enable calculation of conduction velocities.

Blood glucose, cholesterol and triglycerides. Blood samples were centrifuged at room temperature to provide plasma for GOD-PERID glucose assay, CHOD-PAP cholesterol assay and for GPO-PAP triglyceride assay (all test kits from Boehringer Mannheim, Mannheim, Germany) by conventional spectrophotometry.

\section{Statistical analysis}

All data are presented as arithmetic means \pm 1 SD. Statistical comparisons between groups were by one-way analysis of variance (ANOVA); where $F<0.05$ and where there was homogeneity of variances (Cochran's $C$ and Bartlett-Box tests, $p<0.05$ ), groups means were compared using Duncan's multiple range tests. 
Table 1. Comparison of the effects of probucol and vitamin $\mathrm{E}$ in diabetic rats

\begin{tabular}{|c|c|c|c|c|}
\hline & $\begin{array}{l}\text { Control } \\
(n=8)\end{array}$ & $\begin{array}{l}\text { Untreated diabetic } \\
(n=7)\end{array}$ & $\begin{array}{l}\text { Diabetic }+ \text { probucol } \\
(1 \% \text { by diet; } n=7)\end{array}$ & $\begin{array}{l}\text { Diabetic + vitamin } \mathrm{E} \\
(1 \% \text { by diet; } n=8)\end{array}$ \\
\hline Mean daily water intake $(\mathrm{ml})$ & $34 \pm 3$ & $321 \pm 53$ & $341 \pm 49$ & $350 \pm 57$ \\
\hline Mean daily food intake $(\mathrm{g})$ & $35 \pm 4$ & $61 \pm 8$ & $66 \pm 6$ & $67 \pm 7$ \\
\hline Final body weight $(\mathrm{g})$ & $458 \pm 24^{\mathrm{x}}$ & $283 \pm 29 y$ & $296 \pm 25^{y}$ & $328 \pm 43^{y}$ \\
\hline Plasma glucose $(\mathrm{mmol} / \mathrm{l})$ & $8.7 \pm 2^{x}$ & $32.7 \pm 10^{y}$ & $37.3 \pm 7^{y}$ & $41.1 \pm 14^{y}$ \\
\hline Plasma cholesterol (mmol/l) & $1.55 \pm 0.31^{\mathrm{x}}$ & $2.35 \pm 0.59^{y}$ & $2.51 \pm 0.52^{y}$ & $2.33 \pm 0.56^{\mathrm{y}}$ \\
\hline Mean arterial pressure $(\mathrm{mm} \mathrm{Hg})$ & $104 \pm 17$ & $103 \pm 14$ & $98 \pm 11$ & $99 \pm 10$ \\
\hline
\end{tabular}

Data (mean \pm SD) were analysed by ANOVA and betweengroup differences for each variable were tested using Duncan's multiple range test; $\mathrm{a}, \mathrm{b} p<0.05 ; \mathrm{x}, \mathrm{y} p<0.01$. Food and water intake, although expressed per rat, were calculated per cage, so that statistical analysis was not legitimate

Table 2. Comparison of the effects of probucol and evening primrose oil in diabetic rats

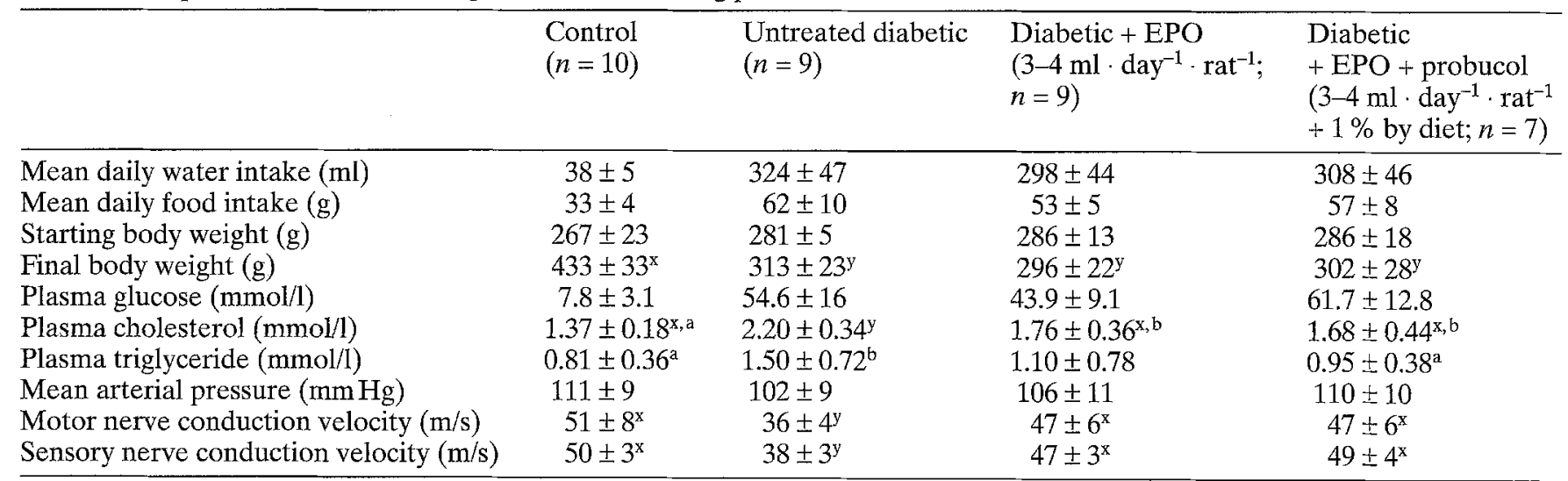

Data (mean \pm SD) were analysed by ANOVA and betweengroup differences for each variable were tested using Duncan's multiple range test; $\mathrm{a}, \mathrm{b} p<0.05 ; \mathrm{x}, \mathrm{y} p<0.01$. Food and

\section{Results}

Animals. All diabetic animals showed persistent hyperphagia, polydipsia and polyuria, which were not modified by the treatments (Tables 1 and 2). Untreated diabetic rats exhibited hyperglycaemia and loss of body weight compared to the non-diabetic control rats and treatment of diabetic rats had no effect on this. Untreated diabetes significantly increased plasma cholesterol and triglyceride concentrations. Neither probucol nor vitamin $\mathrm{E}$ treatment of diabetes affected increased plasma cholesterol concentrations, but probucol alone or in combination with evening primrose oil significantly decreased the raised plasma triglycerides. Vitamin E showed a similar trend towards reduced plasma triglycerides. Evening primrose oil treatment alone or with probucol significantly attenuated the increases in plasma cholesterol, but failed to exert a significant effect on plasma triglycerides when given alone.

Sciatic nerve laser Doppler flux. Figures 1 and 2 show individual animal data points together with group means and standard deviations of sciatic nerve laser water intake, although expressed per rat, were calculated per cage, so that statistical analysis was not legitimate. EPO, Evening primrose oil

Doppler flux in Study 1 and Study 2, respectively. In both studies, sciatic nerve laser Doppler flux was significantly decreased in untreated-diabetic rats compared to corresponding controls. This deficit was attenuated by treatment with vitamin $\mathrm{E}$ or probucol. Evening primrose oil treatment of diabetic rats, either alone or with probucol, increased sciatic nerve laser Doppler flux. In both studies, all rats were normotensive (Tables 1 and 2).

Nerve conduction velocity. Motor and sensory nerve conduction velocities were significantly reduced in untreated diabetic rats compared to control values (Table 2). Evening primrose oil treatment, alone or in combination with probucol, prevented these reductions in conduction velocity.

\section{Discussion}

The data clearly demonstrate that the anti-oxidants probucol or vitamin $\mathrm{E}$ attenuated the depressed sciatic nerve laser Doppler flux in STZ-diabetic rats. Treatment with evening primrose oil alone also im- 
Sciatic nerve laser Doppler flux (arbitrary units)

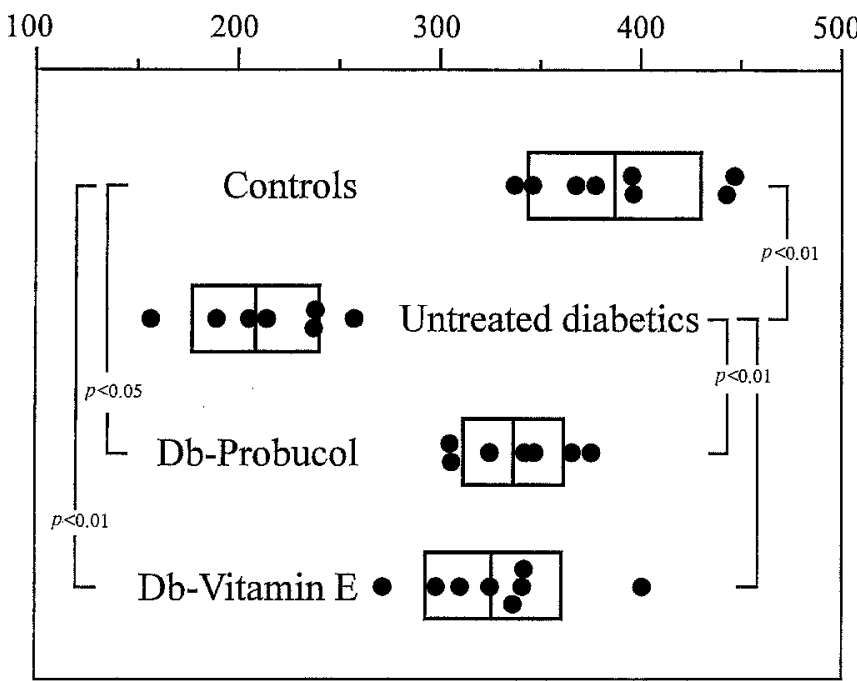

Fig. 1. Sciatic nerve laser Doppler flux data from Study 1. Individual animal values are shown, with boxes denoting SD and arithmetic means in the centre. Statistical comparisons are by one-way ANOVA with Duncan's multiple range tests

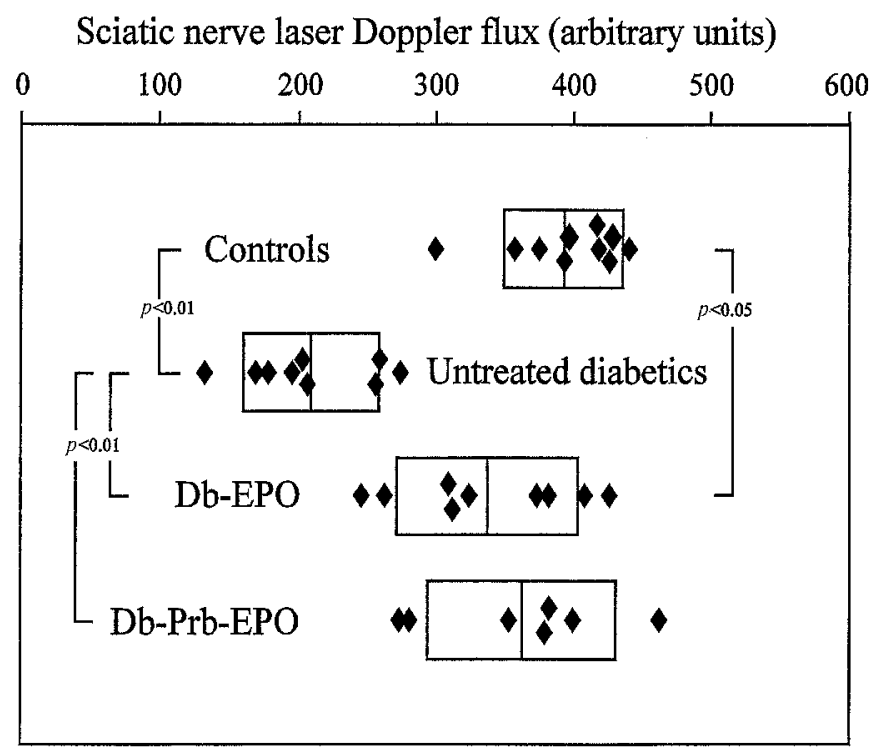

Fig. 2. Sciatic nerve laser Doppler flux data from Study 2. Individual animal values are shown, with boxes denoting SD and arithmetic means in the centre. Statistical comparisons are by one-way ANOVA with Duncan's multiple range tests

proved sciatic nerve laser Doppler flux in diabetic rats. The combined effect of evening primrose oil and probucol was not significantly greater than that of evening primrose oil alone. There was, therefore, little evidence for an additive effect of the two treatments. The data also demonstrate that treatment of diabetic rats with evening primrose oil alone had a marked effect on the motor and sensory nerve conduction velocity deficits. Since these conduction velocities were virtually normalised by evening prim- rose oil alone, there was no scope for demonstration of the additive effects of probucol. The effect of the anti-oxidants alone on nerve conduction velocity was not examined in this study since a recent paper by Cameron et al. [23] has shown that probucol, used in a similar experimental protocol, at the same dose and for a similar duration of treatment to that which we employed, significantly attenuated decreases in motor and sensory nerve conduction velocity. Other studies have also demonstrated similar effects of butylated hydroxytoluene [24] and reduced glutathione [25]. The present work, together with published findings, thus indicate that anti-oxidant treatments can improve peripheral nerve dysfunction and may do so via improvement of nerve perfusion. The effects of treatments occurred in the absence of any changes in the severity of diabetes, as indicated by plasma glucose levels, body weight loss, polyphagia and polydipsia. Deleterious effects of oxidative stress may contribute to decreased endoneurial blood flow via impaired endothelial vasorelaxant effects $[11,20]$. It is also possible that impaired endoneurial perfusion might contribute to reduced clearance of free radicals. Thus, their damaging effects may be self-reinforcing.

Raised levels of lipid peroxidation products as a results of oxidative stress is well documented in clinical diabetes [15] and in STZ-diabetic rats [27]. D- $\alpha$ tocopherol, the most active form of vitamin $\mathrm{E}$, is an anti-oxidant that affects membrane-bound enzyme systems $[28,29]$. This vitamin is a potent modulator of endothelial cell function [30] and previous reports have indicated that $D-\alpha$ tocopherol can restore the prostacyclin/thromboxane $\mathrm{A}_{2}$ balance in diabetic rats [31] and also stimulate the production of prostacyclin by cultured aortic endothelial cells at physiological glucose concentrations [32,33]. Many studies also indicate that vitamin $\mathrm{E}$ content in tissues, especially in platelets, is decreased in diabetic animals [34] and high concentrations of glucose reduce D- $\alpha$ tocopherol binding in tissues [35]. These reports thus strongly suggest that vascular defects in diabetes are related to vitamin $\mathrm{E}$ deficiency, leading to increased free radical damage, the accumulation of lipid peroxides and a decrease in the production of prostacyclin by endothelial cells.

The one striking inconsistency of data between the two studies are the mean plasma triglyceride levels in the untreated diabetic groups. We have examined the raw data but cannot explain the differences, although the values associated with the various treatments were more comparable. Thus, our data are in agreement with published evidence showing that the increased levels of plasma triglyceride, lipid peroxidation products and higher protein glycation in diabetes are markedly attenuated by treatment with probucol or vitamin E $[27,36]$. Probucol is a drug widely used in the treatment of hypercholesterolaemia, 
probably mediated by scavenging superoxide anions, which inhibits effectively the oxidative modification of LDL [37]. However, there was no effect of probucol on the increased plasma cholesterol levels. It has been demonstrated that probucol treatment can reduce serum total cholesterol levels in 4-week STZ-diabetic rats [38] and Barnhart et al. [39] also reported that a $1 \%$ probucol diet is sufficient to reduce plasma cholesterol in the rat. These observations are not supported by our results, although an explanation for this is unclear as the probucol treatment may be considered efficacious due to its functional effects.

We have previously shown that the reduced sciatic nerve laser Doppler flux in diabetic rats indicates possible ischaemia and that a high dose of evening primrose oil has the potential to prevent nerve ischaemia and conduction abnormalities [5, 40]. We also reported that evening primrose oil treatment increases tissue prostacyclin production [9]. These findings are supported by the demonstration that the beneficial effect of evening primrose oil is completely blocked by the co-administration to diabetic rats of flurbibrofen [41] and that nerve function is also restored by a prostacyclin analogue [7]. The present study confirms that a lower dose of evening primrose oil is adequate for efficacy against both nerve ischaemia and conduction defects. Our study also indicates that anti-oxidant properties of the unsaturated fatty acids in evening primrose oil must be considered as components of its effects on peripheral nerves since there was little additive effect of this treatment together with probucol. Detailed examination of its effects on lipid peroxides is warranted.

Acknowledgements. This work was supported by a grant from the William Harvey Research Institute made possible by donations from Ono Pharmaceuticals and Scotia Pharmaceuticals. We are grateful to Dr. D. Horrobin for a gift of evening primrose oil.

\section{References}

1. Tuck RR, Schmelzer JD, Low PA (1984) Endoneurial blood flow and oxygen tension in the sciatic nerves of rats with experimental diabetic neuropathy. Brain 107: 935-950

2. Ram Z, Sadeh M, Walden R, Adar R (1991) Vascular insufficiency quantitatively aggravates diabetic neuropathy. Arch Neurol 48: 1239-1242

3. Monafo WW, Eliasson SG, Shimazaki S, Sugimoto $H$ (1988) Regional blood flow in resting and stimulated sciatic nerve of diabetic rats. Exp Neurol 99: 607-614

4. Cameron NE, Cotter MA, Low PA (1991) Nerve blood flow in early experimental diabetes in rats: relation to conduction deficits. Am J Physiol 261: E1-E8

5. Stevens EJ, Lockett MJ, Carrington AL, Tomlinson DR (1993) Essential fatty acid treatment prevents nerve ischaemia and associated conduction anomalies in rats with experimental diabetes mellitus. Diabetologia 36: 397-401

6. Robertson S, Cameron NE, Cotter MA (1992) The effect of the calcium antagonist nifedipine on peripheral nerve func- tion in streptozotocin-diabetic rats. Diabetologia 35: 11131117

7. Cotter MA, Dines KC, Cameron NE (1993) Prevention and reversal of motor and sensory peripheral nerve conduction abnormalities in streptozotocin-diabetic rats by the prostacyclin analogue iloprost. Naunyn Schmiedebergs Arch Pharmacol 347: 534-540

8. Cameron NE, Cotter MA, Robertson S (1992) Angiotensin converting enzyme inhibition prevents development of muscle and nerve dysfunction and stimulates angiogenesis in streptozotocin-diabetic rats. Diabetologia 35: 12-18

9. Stevens EJ, Carrington AL, Tomlinson DR (1993) Prostacyclin release in experimental diabetes: effects of evening primrose oil. Prostaglandins Leukot Essent Fatty Acids 49: 699-706

10. Oberley LW (1988) Free radicals and diabetes. Free Radiol Biol Med 5: 113-124

11. Pieper GM, Garrett JG (1988) Oxygen free radicals abolish endothelium dependent relaxation in diabetic rat aorta. Am J Physiol 255: H825-H833

12. Jain SK, McVie R, Duett J, Herbst JJ (1989) Erythrocyte membrane lipid peroxidation and glycosylated hemoglobin in diabetes. Diabetes 38: 1539-1543

13. Armstrong D, Al-Awadi F (1991) Lipid peroxidation and retinopathy in streptozotocin-induced diabetes. Free $\mathrm{Ra}$ dic Biol Med 11: 433-436

14. Low PA, Nickander KK (1991) Oxygen free radical effects in sciatic nerve in experimental diabetes. Diabetes 40 : $873-877$

15. Nishigaki I, Hagihara M, Tsunekawa T, Masaki M, Yagi K (1981) Lipid peroxide level of serum lipoprotein fractions of diabetic patients. Biochem Med 25: 373-378

16. Jongkind JF, Verkerk A, Baggen RGA (1989) Glutathione metabolism of human vascular endothelial cells under peroxidative stress. Free Radical Biol Med 7: 507-512

17. Mullarkey CJ, Edelstein D, Brownlee M (1990) Free radical generation by early glycation products: a mechanism for accelerated atherogenesis in diabetes. Biochem Biophys Res Comm 173: 932-939

18. Lyons TJ (1992) Lipoprotein glycation and its metabolic consequences. Diabetes 41 (Suppl 2): 67-73

19. Chisolm GM, Morel DW (1988) Lipoprotein oxidation and cytotoxicity: effect of probucol on streptozotocin-treated rats. Am J Cardiol 62: 20B-26B

20. Pieper GM, Langenstroer P, Gross GJ (1993) Hydroxyl radicals mediate injury to endothelium-dependent relaxation in diabetic rat. Mol Cell Biochem 122: 139-145

21. Tesfamariam B, Cohen RA (1992) Role of superoxide anion and endothelium in the vasoconstrictor action of prostaglandin endoperoxide. Am J Physiol 262: H1915-H1919

22. Tesfamariam B, Cohen RA (1992) Free radicals mediate endothelial cell dysfunction caused by elevated glucose. Am J Physiol 263: H321-H326

23. Cameron NE, Cotter MA, Archibald V, Dines KC, Maxfield EK (1994) Anti-oxidant and pro-oxidant effects on nerve conduction velocity, endoneurial blood flow and oxygen tension in non-diabetic and streptozotocin-diabetic rats. Diabetologia 37: 449-459

24. Cameron NE, Cotter MA, Maxfield EK (1993) Anti-oxidant treatment prevents the development of peripheral nerve dysfunction in streptozotocin-diabetic rats. Diabetologia 36: 299-304

25. Bravenboer B, Kappelle AC, Hamers FPT, Van Buren T, Erkelens DW, Gispen WH (1992) Potential use of glutathione for the prevention and treatment of diabetic neuropathy in the streptozotocin-induced diabetic rat. Diabetologia 35 : $813-817$ 
26. Stevens EJ, Carrington AL, Tomlinson DR (1994) Nerve ischaemia in diabetic rats: time-course of development, effect of insulin treatment plus comparison of streptozotocin and BB models. Diabetologia 37: 43-48

27. Pritchard KA, Patel STJ, Karpen CW, Newman HAI, Pangamala RV (1986) Triglyceride lowering effect of dietary vitamin $E$ in streptozotocin-induced diabetic rats. Increased lipoprotein lipase activity in livers of diabetic rats fed high dietary vitamin E. Diabetes 35: 278-281

28. Tappel AL (1976) Vitamin $E$ and free radical peroxidation of lipids. Ann NY Acad Sci 203: 12-28

29. Bieri JG, Farrell PM (1976) Vitamin E. Vit Horm 34: 31-75

30. Huang N, Lineberg B, Stiner M (1988) Alpha-tocopherol, a potent modulator of endothelial cell function. Thromb Res 50: $547-557$

31. Karpen CW, Pritchard KAJ, Arnold H, Cornwell DG, Pangamala RV (1982) Restoration of prostacyclin/thromboxane $\mathrm{A} 2$ balance in the diabetic rat: influence of dietary vitamin E. Diabetes 31: 947-951

32. Kunusaki M, Umeda F, Inoguchi T, Nawata H (1992) Vita$\min \mathrm{E}$ restores reduced prostacyclin synthesis in aortic endothelial cells cultured with a high concentration of glucose. Metabolism 41: 613-621

33. Umeda F, Kunisaki M, Inoguchi T, Nawata H (1990) Endhanced prostacyclin production by cultured aortic endothelial cells. J Clin Biochem Nutr 8: 175-183

34. Kunisaki M, Umeda F, Inoguchi $T$, Watanabe J, Nawata $H$ (1990) Effect of vitamin E administration of platelet function in diabetes mellitus. Diabetes Res 14: 37-42
35. Kunisaki M, Umeda F, Teruaki Y, Masakado M, Nawata H (1993) High glucose reduces specific binding for D-tocopherol in cultured aortic endothelial cells. Diabetes 42: 1138-1146

36. Ceriello A, Guigliano D, Quatraro A, Donzella A, Dipalo G, Lefebvre P (1991) Vitamin E reduction of protein glycosylation in diabetes: new prospect for prevention of diabetic complications. Diabetes Care 14: 68-72

37. Parthasarthy S, Young SG, Witztum RC (1986) Probucol inhibits oxidative modification of low density lipoprotein. J Clin Invest 77: 641-644

38. Tada H, Oida K, Kutsumi Y, Shimada Y, Nakai T, Miyabo S (1992) Effects of probucol on impaired cardiac performance and lipid metabolism in streptozotocin-induced diabetic rats. J Cardiovasc Pharmacol 20: 179-186

39. Barnhart JW, Sefranka JA, McIntosh DD (1970) Hypocholesterolemic effect of 4,4"-(isopropylidenedithio) bis $(2,6$ di-t-butylphenol) (probucol). Am J Clin Nutr 23: 12291233

40. Lockett MJ, Tomlinson DR (1992) The effects of dietary treatment with essential fatty acids on sciatic nerve conduction and activity of the $\mathrm{Na}^{+} / \mathrm{K}^{+}$pump in streptozotocin-diabetic rats. Br J Pharmac 105: 355-360

41. Cameron NE, Cotter MA, Dines KC, Maxfield EK (1993) Pharmacological manipulation of vascular endothelium function in non-diabetic and streptozotocin-diabetic rats: effects on nerve conduction, hypoxic resistance and endoneurial capillarization. Diabetologia 36: 516-522 